\title{
Predictors of Histological Upgrading at Radical Prostatectomy in Patients With Localised Low- Grade Prostate Cancer and Pretreatment Biparametric MRI
}

Ola Christiansen ( $\boldsymbol{D}$ ola.christiansen@sykehuset-innlandet.no)

Innlandet Hospital Trust

Ola Bratt

Sahlgrenska University Hospital

Øyvind Kirkevold

Innlandet Hospital Trust

Jūratè Šaltytė Benth

University of Oslo

Pathmakulendran Manoharan

Innlandet Hospital Trust

Anders Selnes

Oslo University Hospital

Erik Skaaheim Haug

Vestfold Hospital Trust

Marit Slaaen

Innlandet Hospital Trust

Research Article

Keywords: Histological upgrading, Prostate cancer, Biparametric MRI, Low-grade, Robotic-assisted radical prostatectomy

Posted Date: April 28th, 2021

DOl: https://doi.org/10.21203/rs.3.rs-444072/v1

License: (c) (i) This work is licensed under a Creative Commons Attribution 4.0 International License.

Read Full License 


\section{Abstract \\ Background}

PSA density has previously been identified as a predictor of histological upgrading at radical prostatectomy in men eligible for active surveillance, but how information from pretreatment biparametric magnetic resonance imaging (bpMRI) contributes needs further clarification. The objective of this register-based study was to identify predictors of upgrading at prostatectomy in men with Gleason Grade (GG) group 1 and pretreatment bpMRI.

\section{Methods}

This study included all 130 men with GG 1 cancer who between March 2014 and September 2019 had a robotic-assisted radical prostatectomy (RARP) at Innlandet Hospital Trust and a bpMRI before their diagnostic biopsy. The outcome measure was upgrading from GG 1 on biopsy to GG 2-5 in the radical prostatectomy specimen. Logistic regression models were used to explore predictors for upgrading. Odds ratios (ORs) and confidence intervals (Cls) were calculated. The association between PSA density and the probability of upgrading was assessed after stratifying by Prostate Imaging-Reporting and Data System and Reporting Datasystem (PI-RADS): PI-RADS 1-3 versus 4-5.

\section{Results}

Upgrading was present in $56 \%(73 / 130)$ of the cancers. PSA density was the only significant predictor for upgrading ( $\mathrm{OR}=1.7,95 \% \mathrm{Cl} 1.2 ; 2.5)$. The probability of upgrading was higher for men with a PIRADS 13 than for men with PIRADS $4-5$, but the difference was not statistically significant (adjusted OR $0.4,95 \%$ $\mathrm{Cl} 0.2 ; 1.1, \mathrm{p}=0.082)$. However, among men with PI-RADS 1-3, the probability gradually increased with increasing PSA density $(p=0.036)$. With PI-RADS $4-5$ the probability of upgrading was high over the entire PSA density range.

\section{Conclusion}

PSA density is a clinically important factor to predict upgrading from GG 1 to GG $2-5$ when bpMRI shows no suspicious lesion. In men with a suspicious tumour on bPMRI (PI-RADS 4-5), the probability of an undetected GG 2-5 cancer is high regardless of the PSA density.

\section{Background}

The clinical diversity of localized prostate cancer often makes it difficult to decide how best to treat an individual patient. Clinically significant prostate cancers eventually may cause local symptoms, spread to other organs and lead to death. Opposed to this, insignificant, low-grade, prostate cancers progress over 
decades, or not at all, and the patients die of other causes. Guidelines recommend monitoring men with presumed insignificant prostate cancer rather than treating them (1). Despite this, many men with lowgrade prostate cancer are still offered either radiation therapy or surgery. One reason may be that some men that we presume have a low-grade cancer actually harbour an undetected high-grade cancer and that we have insufficient models to predict this (2).

To aid treatment decisions, prostate cancer is categorised in risk groups, based on serum prostate specific antigen (PSA), histological grade and tumour stage.

The International Society of Urological Pathology (ISUP) recommends that prostate cancer's histology is categorized into five groups (3). There is no consensus on how to define clinically significant prostate cancer, but one common definition is grade group (GG) 2-5 (4). According to this definition, GG 1 (previously named Gleason score 6) represents clinically insignificant cancer, and GG 2-5 represent significant cancer (previously Gleason score 7-10).

Prostate magnetic resonance imaging (MRI) can not only aid prostate cancer diagnostics, but also predict histological grade and may add valuable information about staging (5). Prostate ImagingReporting and Data System (PI-RADS) is the method of choice for evaluation of MRI of the prostate gland $(6,7)$. Although the PI-RAD system is based on multiparametric MRI, it can also be applied on biparametric MRI (8). Biparametric MRI (bpMRI), a protocol without dynamic contrast enhancement, has detection rates comparable with multiparametric MRI, but is less time-consuming and therefore more cost-effective (9).

For men with GG 1 on biopsy, upgrading in the final histology is present in more than one third of the patients (10-12). PSA related to prostate volume (PSA density) is a known predictor for histological upgrading in the prostatectomy specimen $(10,13)$. Furthermore, both PI-RADS score and PSA density prior to initial biopsy and diagnosis can predict the presence of GG 2-5 cancer (14). We are, however, not aware of any studies that have investigated the association between PSA density and the probability of upgrading for men with PI-RAD score $\leq 3$ and PI-RAD score $\geq 4$.

The aim of this study was to identify predictors of upgrading at radical prostatectomy in men with lowrisk cancer who have had a pretreatment bpMRI. In addition, we aimed at exploring the association between PSA density and the probability of upgrading stratified by PI-RADS 1-3 and PI-RADS 4-5.

\section{Methods}

\section{Study design:}

Single-centre, observational study from Innlandet Hospital Trust, Norway.

\section{Study population:}


Between March 2014 and September 2019, 1049 patients underwent robotic-assisted radical prostatectomy (RARP) at Innlandet Hospital Trust. Consenting patients with bpMRI performed at our institution and GG 1 on initial biopsy were included in the study, leaving 130 patients for the analysis. Both patients diagnosed with targeted biopsy and patients diagnosed with systematic biopsies were included.

\section{Clinical and pathological data:}

The clinical data included age, PSA, PSA density, tumour stage on bpMRI, PI-RAD score, GG on pretreatment biopsy, and GG and stage (pT) in the prostatectomy specimen. PSA density was calculated as last available preoperative PSA value divided with the prostate volume as estimated based on MRI measurements (length $\mathrm{x}$ width $\mathrm{x}$ height $\mathrm{x} \pi / 6$ ).

\section{Biparametric MRI:}

All the patients had an MRI before the diagnostic biopsy. MRI was performed with 1.5 Tesla (Phillips Achieva) resolution without endorectal coil. The protocol included T2-weighted and diffusion-weighted images. All MRIs were classified according to PI-RADS (version 1.0 and 2.0). All MRIs were re-evaluated for staging purposes at Innlandet Hospital Trust by an experienced uro-radiologist.

\section{Statistical analysis:}

Clinical characteristics were described by medians, min and max values, means, and standard deviations (SD) for continuous variables, and frequencies and percentages for categorical variables. Unadjusted and adjusted logistic regression models were used to assess the preoperative factors (age, PSA density, PIRADS (dichotomized as 1-3 versus 4-5) and MRI stage (dichotomized as T1-2 versus T3a or T3b) as predictors for the outcome measure histological upgrading to GG 2-5 in the prostatectomy specimen. Odds ratios (ORs) with 95\% confidence intervals (Cls) were calculated. The association between PSA density and the probability of upgrading among patients with PI-RADS 1-3 and PI-RADS 4-5 on MRI was explored by logistic regression model with PSA density, PI-RADS and the interaction between these two. Given the limited sample size and the distribution of the outcome measure, the number of preoperative factors that were included in the regression analysis had to be restricted. We chose to include PSA density in the model, but excluded PSA, as previous studies have unanimously shown that PSA density is the better marker of the two.

All tests were two-sided, and results with $p$-values $<0.05$ were considered statistically significant. Statistical analyses were done with SPSS v26.

\section{Results}

Descriptive statistics of the study population are presented in Table 1. 
Upgrading from GG 1 to GG 2-5 was present in more than half of the patients (56\%). PSA density was the only preoperative factor that significantly predicted upgrading $(\mathrm{OR}=1.7,95 \% \mathrm{Cl} 1.2 ; 2.5$, Table 2$)$. The association between PSA density and the probability of upgrading is illustrated in Fig. 1. More than half of the patients with PSA density above $0.20 \mathrm{ng} / \mathrm{mL} / \mathrm{cm}^{3}$ had their cancer upgraded in the radical prostatectomy specimen. The probability of upgrading was about half as high for men with a PIRADS 13 on MRI as for men with PIRADS $4-5$, but the difference was not statistically significant (adjusted OR $0.4,95 \% \mathrm{Cl} 0.2 ; 1.1, \mathrm{p}=0.082$ ).

For patients with PIRADS $4-5$, the probability of upgrading was high over the entire PSA density range ( $p$ $=0.061$ ), whereas for patients with PI-RADS 1-3 the probability increased from very low for men with low PSA density to high for those with higher PSA density ( $p=0.036$, Fig. 2$)$.

\section{Discussion}

The aim of this study was to identify predictors of upgrading at prostatectomy in men with GG 1 and pretreatment biparametric MRI. PSA density was the only significant predictor for upgrading, but according to exploratory analyses its clinically importance merely applies to patients with a bpMRI and PI-RADS 1-3. For patients with PI-RADS 4-5, the probability of upgrading was high also if their PSA density was low.

Our results regarding the overall impact of PSA density are in line with previously published studies. In a population-based study, approximately half of the men with GG 1 cancer who underwent prostatectomy had adverse pathology (defined as upgrading from GG 1 or upstaging to T3a or T3b) in the prostatectomy specimen (10). Age, PSA, PSA density above $0.15 \mathrm{ng} / \mathrm{ml} / \mathrm{cm}^{3}$, clinical stage T3 and more than $4 \mathrm{~mm}$ cancer length on biopsy predicted adverse pathology. Another study that compared PSA and PSA density as predictors for upgrading after prostatectomy in men with GG 1 concluded that PSA density is a significantly better predictor than PSA (15).

In our study, upgrading was also present in about half of the men, consistent with previous publications $(11,12)$. In a recent study, upgrading from GG 1 to GG $2-5$ was found in $40 \%$ of men with very-low-risk disease and $59 \%$ with low-risk disease (12). The authors recommend, as stated in guidelines, confirmatory testing for patients eligible for active surveillance (repeat biopsy, prostate MRI or genomic markers), as this can identify patients at risk for adverse pathology after surgery.

Our finding that PSA density is of clinical importance mainly for patients with PI-RADS 1-3 on bpMRI, not for patients with PI-RADS 4-5, has, to the best of our knowledge, not been previously reported. It is, however, supported by previous studies showing that the pre-diagnostic probability of significant prostate cancer in patients with PI-RADS 1-3 lesions is associated with PSA density: the risk of harbouring a significant cancer is high if PSA density is above $0.15 \mathrm{ng} / \mathrm{ml} / \mathrm{cm}^{3}(4,14)$.

Our results are clinically important when considering active surveillance. They clearly suggest that men with GG 1 and PI-RADS 4-5 on bpMRI are at great risk of having GG 2-5, regardless of the PSA density. 
Patients should be informed about this and recommended either repeated targeted biopsies or radical treatment. On the other side, PSA density should strongly influence the treatment decision for men with GG 1 and PI-RADS score 1-3. Men with PI-RADS score 1-3 and low PSA density should be advised to start on active surveillance and men with high PSA density to have either a systematic biopsy or treatment. A previous randomised study showed that GG 2-5 cancers are commonly located in the ventral part of the prostate in men with GG 1 on systematic transrectal biopsy and a high PSA density, mandating specific ventral biopsy sampling in this patient group (16)

In our main regression analyses, we found that neither the PI-RADS score nor the MRI tumour stage was associated with upgrading. This is in contrast to two other studies, that reported an association between PI-RADS score and upgrading after prostatectomy in men with GG 1 (17) (18). One of them did, however, include patients with GG 1-5 on biopsy, of which only 58 had GG 1 (18). The other study included only patients with GG 1 and they found that PI-RADS score 4-5 was associated with upgrading (17).

Nevertheless, in our study the confidence intervals were wide, so we cannot exclude that the reason why no significant association was detected could be related to our relatively small sample size.

A strength of our study is that data were prospectively collected. Limitations include the small sample size and the unknown selection process leading to surgery. The latter means that our results are not representative for all patients diagnosed with $G G$ 1, as the patients in our study might have been recommended surgery because of MRI findings, a high PSA density or rising PSA values. Moreover, interrater discrepancy for MRI reading and tumour grading may affect the external validity of our results (19). Prostate MRI is routinely performed before biopsy, and there were several radiologists involved in the PIRADS scoring of the MRI scans. Staging, however, was accomplished by a dedicated MRI uroradiologist with several years of experience. In addition, biparametric MRI is increasingly used, and it is important that studies with bpMRI are done, but it is a limitation that the results are not valid for multiparametric MRI. Another limitation is how the biopsies were taken. This register-based study has recruited patients over several years and the routine for how biopsies are performed has changed over time. Hence, some patients had systematic biopsies, while others are diagnosed after guided biopsies. Furthermore, the histological specimens were not reviewed. This may, however, just as easily be seen as a strength as a limitation. In routine clinical practice, specimens are not reviewed, thus our study and its findings reflects the reality for many patients and clinicians.

\section{Conclusion}

PSA density is a strong predictor for upgrading at radical prostatectomy in men with GG 1 prostate cancer on biopsy only for those with a biparametric MRI categorised as PI-RADS 1-3. Our study suggests that active surveillance is unsuitable for men with a PI-RADS 4-5 tumour and for men with PI-RADS 1-3 and a high PSA density.

\section{Abbreviations}


bpMRI: Biparametric magnetic resonance imaging

GG: Gleason Grade

ISUP: International Society of Urological Pathology

PI-RADS: Prostate Imaging-Reporting and Data System

PSA: Prostate specific antigen

RARP: Robotic-assisted radical prostatectomy

\section{Declarations}

Ethics approval and consent to participate: All patients gave their informed consent to participate. Approval for all parts of the project was applied for to the Regional Committee for Medical Research Ethics, Health Region South-East (REK HS $\varnothing$ - REF 2021/229048), Norway and the Data Protection Official for Research at Innlandet Hospital Trust. The study was performed according to the rules of the Helsinkideclaration. Participation did not inflict upon the participants' treatment and did not imply any health risks or deviation from good clinical practice.

Consent for publication: None

Availability of data and materials: The data are available from the corresponding author on request.

Competing interests: The authors declare that they have no competing interests.

Authors contributions: $\mathrm{OC}, \mathrm{OB}$ and MS contributed to the design and writing the manuscript. JSB performed the statistical analysis and revised the manuscript. PM re-read the MRI images and revised the manuscript. $\varnothing \mathrm{K}, \mathrm{AS}$ and ESH draftet and revised the manuscript. All authors read and approved the final manuscript.

Funding: This study was funded by Innlandet Hospital Trust.

Acknowledgements: Not applicable

\section{References}

1. European Association of Urology (EAU). Prostate Cancer 2021. http://uroweb.org/guideline/prostatecancer/. Assessed 24 March 2021

2. Athanazio D, Gotto G, Shea-Budgell M, Yilmaz A, Trpkov K. Global Gleason grade groups in prostate cancer: concordance of biopsy and radical prostatectomy grades and predictors of upgrade and downgrade. Histopathology. 2017;70(7):1098-106. 
3. Epstein JI, Egevad L, Amin MB, Delahunt B, Srigley JR, Humphrey PA. The 2014 International Society of Urological Pathology (ISUP) Consensus Conference on Gleason Grading of Prostatic Carcinoma: Definition of Grading Patterns and Proposal for a New Grading System. The American journal of surgical pathology. 2016;40(2):244-52.

4. Norris JM, Carmona Echeverria LM, Bott SRJ, Brown LC, Burns-Cox N, Dudderidge T, et al. What Type of Prostate Cancer Is Systematically Overlooked by Multiparametric Magnetic Resonance Imaging? An Analysis from the PROMIS Cohort. European urology. 2020;78(2):163-70.

5. Caglic I, Kovac V, Barrett T. Multiparametric MRI - local staging of prostate cancer and beyond. Radiology and oncology. 2019;53(2):159-70.

6. Weinreb JC, Barentsz JO, Choyke PL, Cornud F, Haider MA, Macura KJ, et al. PI-RADS Prostate Imaging - Reporting and Data System: 2015, Version 2. European urology. 2016;69(1):16-40.

7. Turkbey B, Rosenkrantz AB, Haider MA, Padhani AR, Villeirs G, Macura KJ, et al. Prostate Imaging Reporting and Data System Version 2.1: 2019 Update of Prostate Imaging Reporting and Data System Version 2. European urology. 2019;76(3):340-51.

8. Boesen L, Norgaard N, Logager V, Balslev I, Bisbjerg R, Thestrup KC, et al. Prebiopsy Biparametric Magnetic Resonance Imaging Combined with Prostate-specific Antigen Density in Detecting and Ruling out Gleason 7-10 Prostate Cancer in Biopsy-naive Men. European urology oncology. 2019;2(3):311-9.

9. Boesen L, Norgaard N, Logager V, Balslev I, Bisbjerg R, Thestrup KC, et al. Assessment of the Diagnostic Accuracy of Biparametric Magnetic Resonance Imaging for Prostate Cancer in BiopsyNaive Men: The Biparametric MRI for Detection of Prostate Cancer (BIDOC) Study. JAMA network open. 2018;1(2):e180219.

10. Vellekoop A, Loeb S, Folkvaljon Y, Stattin P. Population based study of predictors of adverse pathology among candidates for active surveillance with Gleason 6 prostate cancer. The Journal of urology. 2014;191(2):350-7.

11. Verep S, Erdem S, Ozluk Y, Kilicaslan I, Sanli O, Ozcan F. The pathological upgrading after radical prostatectomy in low-risk prostate cancer patients who are eligible for active surveillance: How safe is it to depend on bioptic pathology? The Prostate. 2019;79(13):1523-9.

12. Kaye DR, Qi J, Morgan TM, Linsell S, Ginsburg KB, Lane BR, et al. Pathological upgrading at radical prostatectomy for patients with Grade Group 1 prostate cancer: implications of confirmatory testing for patients considering active surveillance. BJU international. 2019;123(5):846-53.

13. Magheli A, Hinz S, Hege C, Stephan C, Jung K, Miller K, et al. Prostate specific antigen density to predict prostate cancer upgrading in a contemporary radical prostatectomy series: a single center experience. The Journal of urology. 2010;183(1):126-31.

14. Distler FA, Radtke JP, Bonekamp D, Kesch C, Schlemmer HP, Wieczorek K, et al. The Value of PSA Density in Combination with PI-RADS ${ }^{\text {TM }}$ for the Accuracy of Prostate Cancer Prediction. The Journal of urology. 2017;198(3):575-82. 
15. Oh JJ, Hong SK, Lee JK, Lee BK, Lee S, Kwon OS, et al. Prostate-specific antigen vs prostate-specific antigen density as a predictor of upgrading in men diagnosed with Gleason 6 prostate cancer by contemporary multicore prostate biopsy. BJU international. 2012;110(11 Pt B):E494-9.

16. Bratt O, Holmberg E, Andrén O, Carlsson S, Drevin L, Johansson E, et al. The Value of an Extensive Transrectal Repeat Biopsy with Anterior Sampling in Men on Active Surveillance for Low-risk Prostate Cancer: A Comparison from the Randomised Study of Active Monitoring in Sweden (SAMS). European urology. 2019;76(4):461-6.

17. Song W, Bang SH, Jeon HG, Jeong BC, Seo SI, Jeon SS, et al. Role of PI-RADS Version 2 for Prediction of Upgrading in Biopsy-Proven Prostate Cancer With Gleason Score 6. Clinical genitourinary cancer. 2018;16(4):281-7.

18. Alqahtani S, Wei C, Zhang Y, Szewczyk-Bieda M, Wilson J, Huang Z, et al. Prediction of prostate cancer Gleason score upgrading from biopsy to radical prostatectomy using pre-biopsy multiparametric MRI PIRADS scoring system. Sci Rep. 2020;10(1):7722.

19. Girometti R, Giannarini G, Greco F, Isola M, Cereser L, Como G, et al. Interreader agreement of PI-RADS v. 2 in assessing prostate cancer with multiparametric MRI: A study using whole-mount histology as the standard of reference. J Magn Reson Imaging. 2019;49(2):546-55.

\section{Tables}

Table 1 Clinical data for patients with GG 1 and GG 2-5 after prostatectomy, N=130 


\begin{tabular}{|c|c|c|c|}
\hline Covariate & Total & $\begin{array}{l}\text { GG } 1 \\
(\mathrm{~N}=57)\end{array}$ & $\begin{array}{l}\text { GG 2-5 } \\
(\mathrm{N}=73)\end{array}$ \\
\hline \multicolumn{4}{|l|}{ Age, years } \\
\hline Median (min-max) & 65 (44-79) & $63(44-76)$ & $66(46-79)$ \\
\hline Mean (SD) & $63.9(6.6)$ & $63.2(6.5)$ & $64.4(6.6)$ \\
\hline \multicolumn{4}{|l|}{$\mathrm{PSA}, \mathrm{ng} / \mathrm{mL}$} \\
\hline Median (min-max) & $10.0(2.2-56.7)$ & $9.7(3.9-20.8)$ & $10.9(2.2-56.7)$ \\
\hline Mean (SD) & $11.2(6.5)$ & $9.7(3.4)$ & $12.4(7.9)$ \\
\hline \multicolumn{4}{|c|}{ PSA density, $\mathrm{ng} / \mathrm{mL} / \mathrm{cm}^{3}$} \\
\hline \multicolumn{4}{|l|}{ Median (min-max) } \\
\hline Mean (SD) & $0.19(0.04-1.22)$ & $0.17(0.06-0.61)$ & $0.22(0.04-1.22)$ \\
\hline $\begin{array}{l}\text { PI-RAD score } \\
\leq 3, \mathrm{n}(\%)\end{array}$ & $0.23(0.15)$ & $0.19(0.09)$ & $0.26(0.18)$ \\
\hline 4 or $5, n(\%)$ & $27(20.8)$ & $16(28.1)$ & $11(15.1)$ \\
\hline \multicolumn{4}{|l|}{ T1-2, n (\%) } \\
\hline T3a, n (\%) & $102(78.5)$ & $48(84.2)$ & $54(74.0)$ \\
\hline T3b, n (\%) & $25(19.2)$ & $9(15.8)$ & $16(21.9)$ \\
\hline \multicolumn{4}{|l|}{$\mathrm{T} 2, \mathrm{n}(\%)$} \\
\hline Т3a, n (\%) & $83(63.8)$ & $42(73.7)$ & $41(56.2)$ \\
\hline \multirow[t]{4}{*}{ T3b, n (\%) } & $44(33.8)$ & $14(24.6)$ & $30(41.1)$ \\
\hline & $3(2.3)$ & $1(1.8)$ & $2(2.7)$ \\
\hline & - & - & - \\
\hline & - & & \\
\hline
\end{tabular}


Table 2 Predictors for upgrading from biopsy GG 1 to GG 2-5 in radical prostatectomy specimens from 130 men, results of unadjusted and adjusted logistic regression

\begin{tabular}{|c|c|c|c|c|}
\hline \multirow[t]{2}{*}{ Covariate } & \multicolumn{2}{|l|}{ Unadjusted model } & \multicolumn{2}{|l|}{ Adjusted model } \\
\hline & OR $(95 \% \mathrm{Cl})$ & P-value & OR $(95 \% \mathrm{Cl})$ & P-value \\
\hline Age & $1.03(0.98 ; 1.08)$ & 0.308 & $1.01(0.96 ; 1.07)$ & 0.688 \\
\hline PSA density ${ }^{1}$ & $1.65(1.15 ; 2.36)$ & 0.006 & $1.72(1.19 ; 2.49)$ & 0.004 \\
\hline \multicolumn{5}{|l|}{ PI-RADS } \\
\hline $1-3$ & $0.46(0.19 ; 1.08)$ & 0.073 & $0.43(0.17 ; 1.11)$ & 0.082 \\
\hline 4 or 5 - ref. & 1 & & 1 & \\
\hline \multicolumn{5}{|c|}{ Tumour stage on MRI } \\
\hline T2 - ref. & 1 & & 1 & \\
\hline T3a+T3b & $1.88(0.78 ; 4.5)$ & 0.163 & $1.75(0.69 ; 4.4)$ & 0.240 \\
\hline
\end{tabular}

${ }^{1}$ OR for $0.1 \mathrm{ng} / \mathrm{ml} / \mathrm{cm}^{3}$ increments

Figures 


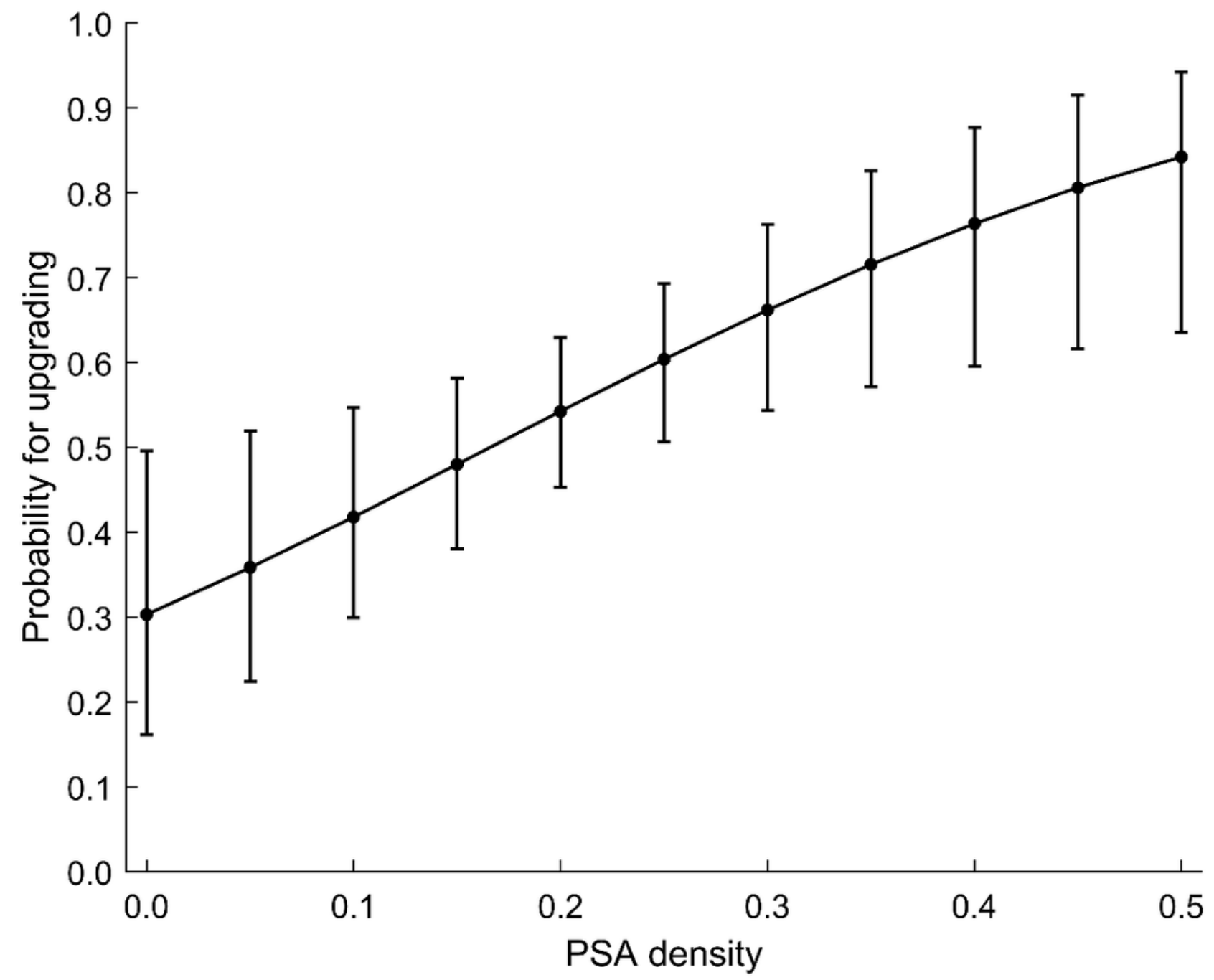

Figure 1

The association between PSA density and the probability for upgrading from biopsy GG 1 to GG 2-5 in radical prostatectomy specimens from 130 men. 


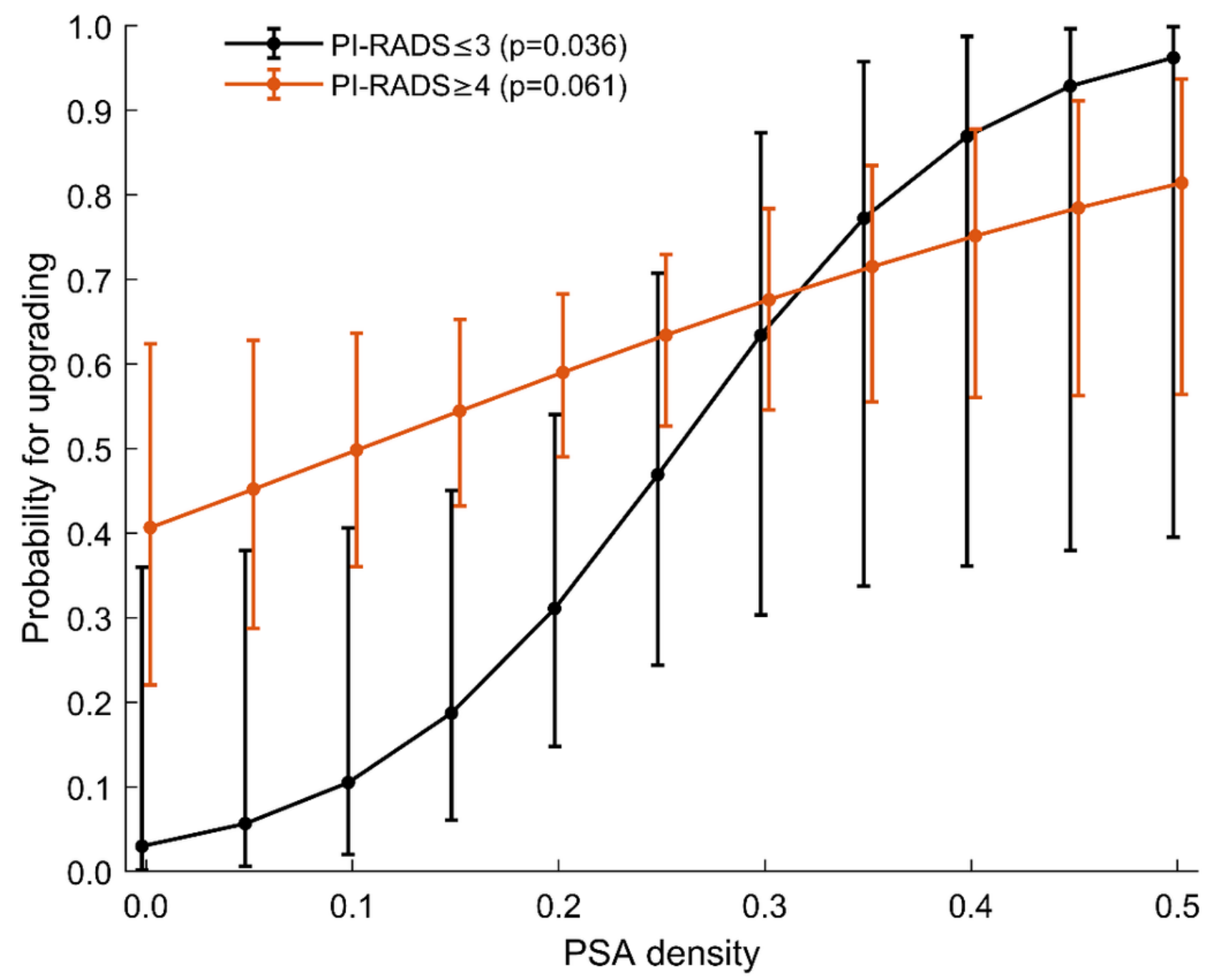

Figure 2

The association between PSA density and the probability of upgrading from biopsy GG 1 to GG 2-5 in the radical prostatectomy specimen stratified by PI-RADS 1-3 and PI-RADS 4-5. 